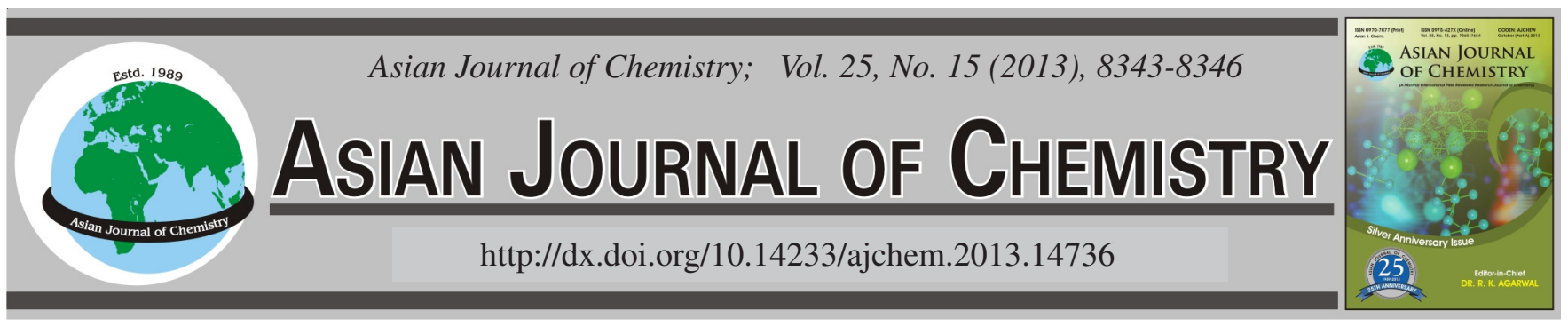

\title{
Titrimetric Analysis of Some Antituberculous Drugs Using Ammonium Hexanitratocerate(IV) Reagent
}

\author{
VinOd Kumar, Brijesh K. Singh and I.C. ShuKLA*
}

Department of Chemistry, University of Allahabad, Allahabad-211 002, India

*Corresponding author: Tel: +91 532 2506858; E-mail: prof.icshukla@ gmail.com

\begin{abstract}
In the present paper we have reported a simple and convenient titrimetric method for determination of antituberculous drugs e.g., cycloserine, ethambutol, isoniazid, pyrazinamide and rifampicin in pure form and in their pharmaceutical preparations such as MD-serine (Tab), pamcerin (Tab), mymbutol (Tab), zytham (Tab), ipcazide (Inj), isokin (Tab), zypra (Tab), P-Zide (Tab), R-Cinex (Tab) and zucox (Tab) with ammonium hexanitratocerate(IV) reagent. It is a versatile oxidizing agent of cerium(IV) and is being widely used as an oxidant for several classes of organic compounds. During estimation it was noted that the excipients present in pharmaceutical preparations do not interfere. The value of percentage error, coefficient of variation (CV) and standard deviation (SD) prove the method to be precise and reproducible. To establish authenticity of the method, recovery experiments were also carried out by standard drug addition method.
\end{abstract}

Key Words: Antituberculous, Ammonium hexanitratocerate(IV), Oxidizing agent, Titration.

ᄂ - - - - - - - - - - - - - - - - - - - - - - - -

\section{INTRODUCTION}

A variety of compounds containing cerium(IV) have proved to be versatile reagents capable of oxidizing almost every oxidizable functional group ${ }^{1}$. Extensive work has lead to the development of a good number of such oxidants like cerium mischmetal ${ }^{2}$, cerium ammonium sulphate ${ }^{3,4}$, cerium sulphate $^{5,6}$, cerium nitrate ${ }^{7}$, cerium fluoride ${ }^{8}$, cerium chloride ${ }^{9,10}$, cerium oxide ${ }^{11,12}$, cerium metal ${ }^{13}$, cerium carbonate ${ }^{14}$ and cerium acetate ${ }^{15}$. There is no general method reported in Indian Pharmacopoeia ${ }^{16}$ for the estimation of all antituberculous drugs. They have reported determination of cycloserine by liquid chromatography, ethambutol and isoniazid by thin layer chromatography while pyrazinamide and rifampicin by infrared absorption spectroscopy in Indian pharmacopoeia.

Industrial demands have led many workers to search for more ideal oxidants with a number of specifications including: lower cost, higher yields, better selectivity, milder neutral conditions, easier preparations, high solubility, less toxicity and short reaction times. Among the above mentioned reagents ${ }^{2-16}$, ammonium hexanitratocerate(IV) has an edge over others for rendering easy manipulation and sharp end points. Therefore we have selected ammonium hexanitratocerate (AHC) as an oxidizing reagent for our study. In the present paper, we described a simple and convenient method for the determination of above antituberculous drugs with ammonium hexanitratocerate(IV) reagent.

\section{EXPERIMENTAL}

Ammonium hexanitratocerate solution $(0.1 \mathrm{M}): 13.70 \mathrm{~g}$ of ammonium hexanitratocerate(IV) was weighed accurately and dissolved in $0.5 \mathrm{~N}$ nitric acid in a $250 \mathrm{~mL}$ volumetric flask.

Ferrous ammonium sulphate solution $(0.025 \mathrm{M})$ : $2.4508 \mathrm{~g}$ of ferrous ammonium sulphate was accurately weighed and dissolved in distilled water in the presence of $10 \mathrm{~mL}$ of $4 \mathrm{M} \mathrm{H}_{2} \mathrm{SO}_{4}$ in a $250 \mathrm{~mL}$ volumetric flask.

Ferroin indicator $(\mathbf{0 . 0 0 1} \mathbf{M})$ : Solution was prepared by diluting $0.025 \mathrm{M}$ ferroin (1,10-phenanthroline ferrous sulphate complex) solution with distilled water.

Sulphuric acid solution (4 M): Solutions were prepared by diluting concentrated sulphuric acid (37N AnalaR, Merck) with distilled water.

Sample solution (1 $\mathbf{~ m g / m L ) : ~ A c c u r a t e l y ~ w e i g h e d ~ ( 1 0 0 ~}$ $\mathrm{mg}$ ) pure samples as well as pharmaceutical preparation of cycloserine, ethambutol, isoniazid and pyrazinamide were dissolve in min amount of cold distilled water and rifampicin was in hot distilled water in a $100 \mathrm{~mL}$ volumetric flask and then made up to the mark with distilled water to give a concentration of $1 \mathrm{mg} / \mathrm{mL}$. While diluting the solution every care was taken to keep the solution homogeneous.

Tablet solution: Twenty tablets of a particular sample were crushed to a fine powder and powder equivalent to $100 \mathrm{mg}$ of sample was taken in $100 \mathrm{~mL}$ volumetric flask and dissolved similarly. No residue was noted in any of the samples. 
Injection solution: As described above the contents of the injection equivalent to $100 \mathrm{mg}$ of the pure sample were taken and dissolved as usual to get a concentration of $1 \mathrm{mg} / \mathrm{mL}$.

Procedure: Aliquots containing 1-5 mg of the samples were taken in $100 \mathrm{~mL}$ stoppered conical flask followed by the addition of $5 \mathrm{~mL}$ ammonium hexanitratocerate(IV) reagent. The reaction mixture was shaken well and allowed to react for required reaction time (10-15 $\mathrm{min})$ at room temperature $(25-$ $30^{\circ} \mathrm{C}$ ). After the reaction was over it was quenched by adding $10 \mathrm{~mL}$ of $4 \mathrm{M}$ sulphuric acid. The unconsumed $\mathrm{Ce}(\mathrm{IV})$ was titrated against $0.025 \mathrm{M}$ ferrous ammonium sulphate solution using two drops of ferroin indicator $(0.001 \mathrm{M})$. A blank experiment was also performed under identical conditions using all the reagents except the sample. The amount of the sample was calculated by the following expression. On the basis of percentage error the value of SD and $\mathrm{CV}$ were also calculated (Table-1). In the case of pharmaceutical preparation the same procedure was applied.

Calculation: For each experiment, the amount of the compound was calculated by following expression:

$$
\text { mg of sample }=\frac{M \times N(B-S)}{n}
$$

\begin{tabular}{|c|c|c|c|c|c|c|c|c|c|}
\hline \multicolumn{10}{|c|}{$\begin{array}{l}\text { TABLE-1 } \\
\text { DETERMINATION OF SOME ANTITUBERCULOUS DRUGS IN PURE FORM AND IN THEIR } \\
\text { PHARMACEUTICAL PREPARATIONS WITH }(0.1 \mathrm{M}) \text { CERIC AMMONIUM NITRATE REAGENT }\end{array}$} \\
\hline $\begin{array}{l}\text { S. } \\
\text { No. }\end{array}$ & Sample & $\begin{array}{l}\text { Aliquots } \\
\text { taken }(\mathrm{mL})\end{array}$ & $\begin{array}{c}\text { Amount } \\
\text { present* (mg) }\end{array}$ & $\begin{array}{l}\text { Reaction } \\
\text { time (min) }\end{array}$ & Molecularity & $\begin{array}{l}\text { Amount obtained by } \\
\text { calculation** }(\mathrm{mg})\end{array}$ & $\begin{array}{l}\text { Error } \\
(\%)\end{array}$ & SD & $\mathrm{CV}$ \\
\hline \multirow{3}{*}{1} & \multirow{3}{*}{$\begin{array}{l}\text { Cycloserine } \\
\text { (Pure Sample) }\end{array}$} & 1.00 & 0.992 & 15 & 6 & 0.982 & -1.01 & 0.0026 & 0.2648 \\
\hline & & 3.00 & 2.976 & 15 & 6 & 2.954 & -0.74 & 0.0033 & 0.1117 \\
\hline & & 5.00 & 4.960 & 15 & 6 & 4.939 & -0.42 & 0.0042 & 0.0850 \\
\hline \multirow{3}{*}{ A } & \multirow{3}{*}{$\begin{array}{c}\text { MD-serine } \\
\text { (Tab) } \\
\text { M.H.Care }\end{array}$} & 1.00 & 0.943 & 15 & 6 & 0.933 & -1.06 & 0.0022 & 0.2358 \\
\hline & & 3.00 & 2.829 & 15 & 6 & 2.808 & -0.74 & 0.0036 & 0.1282 \\
\hline & & 5.00 & 4.715 & 15 & 6 & 4.691 & -0.51 & 0.0044 & 0.0938 \\
\hline \multirow{3}{*}{ B } & Pamcerin (Tab) & 1.00 & 0.953 & 15 & 6 & 0.943 & -1.05 & 0.0044 & 0.4666 \\
\hline & Samarth & 3.00 & 2.859 & 15 & 6 & 2.840 & -0.66 & 0.0033 & 0.1162 \\
\hline & Pharma & 5.00 & 4.765 & 15 & 6 & 4.742 & -0.48 & 0.0022 & 0.0464 \\
\hline \multirow{3}{*}{2} & \multirow{3}{*}{$\begin{array}{l}\text { Ethambutol } \\
\text { (pure Sample) }\end{array}$} & 1.00 & 0.982 & 15 & 6 & 0.972 & -1.02 & 0.0063 & 0.6481 \\
\hline & & 3.00 & 2.946 & 15 & 6 & 2.924 & -0.75 & 0.0042 & 0.1436 \\
\hline & & 5.00 & 4.910 & 15 & 6 & 4.884 & -0.53 & 0.0030 & 0.0614 \\
\hline \multirow{3}{*}{ A } & \multirow{3}{*}{$\begin{array}{l}\text { Mymbutol } \\
\text { (Tab) Wyeth }\end{array}$} & 1.00 & 0.946 & 15 & 6 & 0.938 & -1.05 & 0.0026 & 0.2772 \\
\hline & & 3.00 & 2.844 & 15 & 6 & 2.824 & -0.70 & 0.0055 & 0.1948 \\
\hline & & 5.00 & 4.740 & 15 & 6 & 4.715 & -0.53 & 0.0042 & 0.0891 \\
\hline \multirow{3}{*}{ B } & \multirow{3}{*}{$\begin{array}{l}\text { Zytham (Tab) } \\
\text { Zy. Cadila }\end{array}$} & 1.00 & 0.937 & 15 & 6 & 0.927 & -1.07 & 0.0042 & 0.4531 \\
\hline & & 3.00 & 2.811 & 15 & 6 & 2.793 & -0.64 & 0.0063 & 0.2256 \\
\hline & & 5.00 & 4.685 & 15 & 6 & 4.663 & -0.47 & 0.0072 & 0.1544 \\
\hline \multirow{3}{*}{3} & \multirow{3}{*}{$\begin{array}{l}\text { Isoniazid (pure } \\
\text { sample) }\end{array}$} & 1.00 & 0.976 & 15 & 6 & 0.966 & -1.02 & 0.0022 & 0.2277 \\
\hline & & 3.00 & 2.952 & 15 & 6 & 2.907 & -0.72 & 0.0013 & 0.0447 \\
\hline & & 5.00 & 4.880 & 15 & 6 & 4.853 & -0.55 & 0.0035 & 0.0721 \\
\hline \multirow{3}{*}{ A } & \multirow{3}{*}{$\begin{array}{l}\text { Ipcazide (Inj) } \\
\text { IPCA }\end{array}$} & 1.00 & 0.926 & 15 & 6 & 0.916 & -1.08 & 0.0018 & 0.1965 \\
\hline & & 3.00 & 2.778 & 15 & 6 & 2.755 & -0.83 & 0.0026 & 0.0944 \\
\hline & & 5.00 & 4.630 & 15 & 6 & 4.601 & -0.63 & 0.0038 & 0.0826 \\
\hline \multirow{3}{*}{ B } & \multirow{3}{*}{$\begin{array}{l}\text { Isokin (Tab) } \\
\text { Pfizer }\end{array}$} & 1.00 & 0.906 & 15 & 6 & 0.896 & -1.10 & 0.0031 & 0.3460 \\
\hline & & 3.00 & 2.718 & 15 & 6 & 2.698 & -0.74 & 0.0028 & 0.1038 \\
\hline & & 5.00 & 4.530 & 15 & 6 & 4.506 & -0.53 & 0.0023 & 0.0510 \\
\hline \multirow{3}{*}{4} & \multirow{3}{*}{$\begin{array}{l}\text { Pyrazinamide } \\
\text { (pure sample) }\end{array}$} & 1.00 & 0.954 & 10 & 4 & 0.944 & -1.05 & 0.0056 & 0.5932 \\
\hline & & 3.00 & 2.862 & 10 & 4 & 2.840 & -0.76 & 0.0045 & 0.1585 \\
\hline & & 5.00 & 4.770 & 10 & 4 & 4.745 & -0.52 & 0.0040 & 0.0843 \\
\hline \multirow{3}{*}{ A } & & 1.00 & 0.909 & 10 & 4 & 0.900 & -0.99 & 0.0070 & 0.7778 \\
\hline & Zypra (Tab) & 3.00 & 2.727 & 10 & 4 & 2.707 & -0.73 & 0.0048 & 0.1773 \\
\hline & & 5.00 & 4.545 & 10 & 4 & 4.522 & -0.51 & 0.0049 & 0.1084 \\
\hline & & 1.00 & 0.913 & 10 & 4 & 0.904 & -1.10 & 0.0041 & 0.4535 \\
\hline B & P-Zide (Tab) & 3.00 & 2.739 & 10 & 4 & 2.719 & -0.73 & 0.0028 & 0.1030 \\
\hline & & 5.00 & 4.565 & 10 & 4 & 4.543 & -0.48 & 0.0037 & 0.0814 \\
\hline & & 1.00 & 0.986 & 10 & 4 & 0.976 & -1.02 & 0.0022 & 0.2254 \\
\hline 5 & $\begin{array}{l}\text { Rifampicin } \\
\text { (pure sample) }\end{array}$ & 3.00 & 2.958 & 10 & 4 & 2.934 & -0.81 & 0.0014 & 0.0477 \\
\hline & & 5.00 & 4.930 & 10 & 4 & 4.901 & -0.59 & 0.0016 & 0.0326 \\
\hline & & 1.00 & 0.927 & 10 & 4 & 0.917 & -1.08 & 0.0062 & 0.6761 \\
\hline A & R-Cinex (Tab) & 3.00 & 2.781 & 10 & 4 & 2.761 & -0.72 & 0.0042 & 0.1521 \\
\hline & & 5.00 & 4.635 & 10 & 4 & 4.617 & -0.39 & 0.0036 & 0.0732 \\
\hline & & 1.00 & 0.898 & 10 & 4 & 0.889 & -1.00 & 0.0032 & 0.3600 \\
\hline B & Zucox (Tab) & 3.00 & 2.694 & 10 & 4 & 2.676 & -0.67 & 0.0025 & 0.0934 \\
\hline & USK Pnarma & 5.00 & 4.490 & 10 & 4 & 4.470 & -0.45 & 0.0039 & 0.0872 \\
\hline
\end{tabular}

Tab $=$ Tablet, Inj $=$ Injection, $*$ In each sample nine estimations were done, $* *$ Average of nine determinations. 
where, $\mathrm{M}=$ molecular weight of sample, $\mathrm{N}=$ normality of ferrous ammonium sulphate solution, $\mathrm{B}=$ volume of ferrous ammonium sulphate for blank, $\mathrm{S}=$ volume of ferrous ammonium sulphate for sample, $\mathrm{n}=$ stoichiometry of the reaction.

For testing quantitative validity of the recommended method, standard deviation (SD) and coefficient of variation (CV) were also calculated for each sample size. At least nine determinations were carried out and the average results were noted. To justify the validity of the proposed method, recovery experiments were carried out by the standard drug addition method. A known amount of the pure compound was taken and to this, varying amounts of the pharmaceutical preparations of the same compounds were added. The total amount of the sample was found by the usual method.

$$
\text { Recovery }(\%)=\frac{\mathrm{N}\left(\sum \mathrm{XY}\right)-\left(\sum \mathrm{X}\right)\left(\sum \mathrm{Y}\right)}{\mathrm{N}\left(\sum \mathrm{X}^{2}\right)-\left(\sum \mathrm{X}\right)^{2}} \times 100
$$

where, $\mathrm{N}=\Sigma \mathrm{N}=$ total number of observations, $\mathrm{X}=$ amount of drug added, $\mathrm{Y}=$ amount of drug obtained by calculation.
The determinations were done with varying sample size (i.e., 1-10 mg) but for convenience, results have been shown only with 1, 3 and $5 \mathrm{mg}$ sample size (Table-1).

For every sample of antituberculous drugs $e . g$., cycloserine, ethambutol, isoniazid, pyrazinamide and rifampicin, recovery experiment were also done by standard drug addition method and the results were reported (Tables 2-6).

Standard deviation (SD) was calculated by the expression given below:

$$
\mathrm{SD}=\sqrt{\frac{\left(\mathrm{X}_{1}-\overline{\mathrm{X}}\right)^{2}+\left(\mathrm{X}_{2}-\overline{\mathrm{X}}\right)^{2} \ldots\left(\mathrm{X}_{\mathrm{n}}-\overline{\mathrm{X}}\right)^{2}}{(\mathrm{n}-1)}}
$$

On the basis of the value of SD, the value of coefficient of variation $(\mathrm{CV})$ was calculated by the following expression.

$$
\mathrm{CV}=\frac{\mathrm{SD} \times 100}{\overline{\mathrm{X}}}
$$

where, $\overline{\mathrm{X}}=$ average value of amount obtained by calculations, $X_{1}, X_{2} \ldots X_{n}=$ amount obtained by calculations in different

\begin{tabular}{|c|c|c|c|c|c|c|c|c|}
\hline \multicolumn{9}{|c|}{$\begin{array}{c}\text { TABLE-3 } \\
\text { RECOVERY STUDIES OF ETHAMBUTOL BY STANDARD DRUG ADDITION METHC }\end{array}$} \\
\hline $\begin{array}{l}\text { S. } \\
\text { No. }\end{array}$ & $\begin{array}{l}\text { Number of } \\
\text { observations } \\
\text { (N) }\end{array}$ & $\begin{array}{c}\text { Amount } \\
\text { present } \\
(\text { Pure })(\mathrm{mg})\end{array}$ & $\begin{array}{l}\text { Amount of } \\
\text { drug added } \\
\text { (mg) X }\end{array}$ & $\begin{array}{l}\text { Total amount of } \\
\text { drug obtained by } \\
\text { calculation }(\mathrm{mg})\end{array}$ & $\begin{array}{c}\text { Amount of drug } \\
\text { obtained by } \\
\text { calculation }(\mathrm{mg}) \mathrm{Y}\end{array}$ & $\mathrm{XY}$ & $X^{2}$ & $\begin{array}{c}\text { Recovery } \\
(\%)\end{array}$ \\
\hline 1 & 3 & 0.982 & 0.959 & 1.962 & 0.968 & 0.928 & 0.920 & \multirow{5}{*}{99.47} \\
\hline 2 & 3 & 0.982 & 1.982 & 2.970 & 1.986 & 3.936 & 3.928 & \\
\hline 3 & 3 & 0.982 & 2.988 & 3.983 & 2.957 & 8.812 & 8.928 & \\
\hline 4 & 3 & 0.982 & 3.970 & 4.975 & 3.961 & 15.725 & 15.761 & \\
\hline & $\Sigma \mathrm{N}=12$ & - & $\Sigma X=9.899$ & - & $\Sigma \mathrm{Y}=9.872$ & $\Sigma X Y=29.401$ & $\Sigma \mathrm{X}^{2}=29.537$ & \\
\hline
\end{tabular}
observations, $\mathrm{n}=$ number of observations.

\begin{tabular}{|c|c|c|c|c|c|c|c|c|}
\hline \multicolumn{9}{|c|}{$\begin{array}{c}\text { TABLE-4 } \\
\text { RECOVERY STUDIES OF ISONIAZID BY STAND }\end{array}$} \\
\hline $\begin{array}{l}\text { S. } \\
\text { No. }\end{array}$ & $\begin{array}{l}\text { Number of } \\
\text { observations } \\
\text { (N) }\end{array}$ & $\begin{array}{c}\text { Amount } \\
\text { present } \\
\text { (Pure) }(\mathrm{mg})\end{array}$ & $\begin{array}{l}\text { Amount of } \\
\text { drug added } \\
\text { (mg) X }\end{array}$ & $\begin{array}{l}\text { Total amount of } \\
\text { drug obtained by } \\
\text { calculation (mg) }\end{array}$ & $\begin{array}{c}\text { Amount of drug } \\
\text { obtained by } \\
\text { calculation (mg) Y }\end{array}$ & $X Y$ & $X^{2}$ & $\begin{array}{c}\text { Recovery } \\
(\%)\end{array}$ \\
\hline 1 & 3 & 0.976 & 0.985 & 1.975 & 0.993 & 0.978 & 0.970 & \multirow{5}{*}{99.72} \\
\hline 2 & 3 & 0.976 & 1.969 & 2.964 & 1.971 & 3.881 & 3.877 & \\
\hline 3 & 3 & 0.976 & 2.972 & 3.980 & 2.963 & 8.806 & 8.833 & \\
\hline 4 & 3 & 0.976 & 3.986 & 4.987 & 3.972 & 15.832 & 15.888 & \\
\hline & $\Sigma \mathrm{N}=12$ & - & $\Sigma X=9.912$ & - & $\Sigma Y=9.899$ & $\Sigma X Y=29.497$ & $\Sigma X^{2}=29.568$ & \\
\hline
\end{tabular}

\begin{tabular}{ccccccccc}
\multicolumn{10}{c}{ TABLE-2 } \\
\hline $\begin{array}{c}\text { RECOVERY STUDIES OF CYCLOSERINE BY STANDARD DRUG ADDITION METHOD } \\
\text { No. }\end{array}$ & $\begin{array}{c}\text { Number of } \\
\text { observations } \\
(\mathrm{N})\end{array}$ & $\begin{array}{c}\text { Amount } \\
\text { present } \\
(\text { Pure })(\mathrm{mg})\end{array}$ & $\begin{array}{c}\text { Amount of } \\
\text { drug added } \\
(\mathrm{mg}) \mathrm{X}\end{array}$ & $\begin{array}{c}\text { Total amount of } \\
\text { drug obtained by } \\
\text { calculation }(\mathrm{mg})\end{array}$ & $\begin{array}{c}\text { Amount of drug } \\
\text { obtained by } \\
\text { calculation }(\mathrm{mg}) \mathrm{Y}\end{array}$ & $\mathrm{XY}$ & $\mathrm{X}^{2}$ & $\begin{array}{c}\text { Recovery } \\
(\%)\end{array}$ \\
\hline 1 & 3 & 0.992 & 0.980 & 1.972 & 0.982 & 0.962 & 0.960 & \\
2 & 3 & 0.992 & 1.983 & 2.962 & 1.968 & 3.903 & 3.932 \\
3 & 3 & 0.992 & 2.970 & 3.986 & 2.969 & 8.818 & 8.821 & 99.50 \\
4 & 3 & 0.992 & 3.973 & 4.968 & 3.945 & 15.673 & 15.785 \\
\hline & $\Sigma \mathrm{N}=12$ & - & $\Sigma \mathrm{X}=9.906$ & - & $\Sigma \mathrm{Y}=9.864$ & $\Sigma \mathrm{XY}=29.356$ & $\Sigma \mathrm{X}^{2}=29.498$ \\
\hline
\end{tabular}

\begin{tabular}{ccccccccc}
\multicolumn{10}{c}{ TABLE-5 } \\
\hline $\begin{array}{c}\text { R. } \\
\text { No. }\end{array}$ & $\begin{array}{c}\text { Number of } \\
\text { observations } \\
(\mathrm{N})\end{array}$ & $\begin{array}{c}\text { Amount } \\
\text { present } \\
(\text { Pure })(\mathrm{mg})\end{array}$ & $\begin{array}{c}\text { Amount of } \\
\text { drug added } \\
(\mathrm{mg}) \mathrm{X}\end{array}$ & $\begin{array}{c}\text { Total amount of } \\
\text { drug obtained by } \\
\text { calculation }(\mathrm{mg})\end{array}$ & $\begin{array}{c}\text { Amount of drug } \\
\text { obtained by } \\
\text { calculation }(\mathrm{mg}) \mathrm{Y}\end{array}$ & $\mathrm{XY}$ & $\mathrm{X}^{2}$ & $\begin{array}{c}\text { Recovery } \\
(\%)\end{array}$ \\
\hline 1 & 3 & 0.954 & 0.978 & 1.978 & 0.995 & 0.973 & 0.956 & \\
2 & 3 & 0.954 & 1.985 & 2.987 & 1.951 & 3.873 & 3.940 \\
3 & 3 & 0.954 & 2.980 & 3.964 & 2.969 & 8.848 & 8.880 & 99.62 \\
4 & 3 & 0.954 & 3.965 & 4.972 & 3.958 & 15.693 & 15.721 \\
\hline & $\Sigma \mathrm{N}=12$ & - & $\Sigma \mathrm{X}=9.908$ & - & $\Sigma \mathrm{Y}=9.873$ & $\Sigma \mathrm{XY}=29.387$ & $\Sigma \mathrm{X}^{2}=29.497$ & \\
\hline
\end{tabular}


TABLE-6

RECOVERY STUDIES OF RIFAMPICIN BY STANDARD DRUG ADDITION METHOD

\begin{tabular}{ccccccccc}
\hline $\begin{array}{c}\text { S. } \\
\text { No. }\end{array}$ & $\begin{array}{c}\text { Number of } \\
\text { observations } \\
(\mathrm{N})\end{array}$ & $\begin{array}{c}\text { Amount } \\
\text { present } \\
(\text { Pure })(\mathrm{mg})\end{array}$ & $\begin{array}{c}\text { Amount of } \\
\text { drug added } \\
(\mathrm{mg}) \mathrm{X}\end{array}$ & $\begin{array}{c}\text { Total amount of } \\
\text { drug obtained by } \\
\text { calculation }(\mathrm{mg})\end{array}$ & $\begin{array}{c}\text { Amount of drug } \\
\text { obtained by } \\
\text { calculation }(\mathrm{mg}) \mathrm{Y}\end{array}$ & $\mathrm{XY}$ & $\mathrm{X}^{2}$ & $\begin{array}{c}\text { Recovery } \\
(\%)\end{array}$ \\
\hline 1 & 3 & 0.986 & 0.982 & 1.976 & 0.996 & 0.978 & 0.964 & \\
2 & 3 & 0.986 & 1.974 & 2.964 & 1.970 & 3.889 & 3.897 & \\
3 & 3 & 0.986 & 2.963 & 3.982 & 2.871 & 8.507 & 8.779 & 99.15 \\
4 & 3 & 0.986 & 3.966 & 4.981 & 3.972 & 15.753 & 15.729 \\
\hline & $\Sigma \mathrm{N}=12$ & - & $\Sigma \mathrm{X}=9.885$ & - & $\Sigma \mathrm{Y}=9.809$ & $\Sigma \mathrm{XY}=29.127$ & $\Sigma \mathrm{X}^{2}=29.369$ \\
\hline
\end{tabular}

\section{RESULTS AND DISCUSSION}

It was found that the stoichiometric ratio between ammonium hexanitratocerate(IV) reagent and antituberculous drugs varies. Different stoichiometric ratio is obtained in different antituberculous drugs such as cycloserine (1:6), ethambutol (1:6), isoniazid (1:6), pyrazinamide (1:4) and rifampicin (1:4) in pure form and in their pharmaceutical preparations. The ratio remains constant even under varying reaction conditions i.e., change in reaction time, concentration of the reagent, reaction medium, reaction temperature etc. It was observed that all the compounds studied need $15 \mathrm{~min}$ to complete the reaction except pyrazinamide and rifampicin which require only 10 min. By the allowing more reaction time (more than 10-15 $\mathrm{min}$ ) there is no improvement in the results. A lesser reaction time (less than $10 \mathrm{~min}$ ) than the described limit gives higher percentage of error because of incomplete reaction. The effect of concentration of ammonium hexanitratocerate reagent (0.01-0.16 M) was also studied and it was found that the recommended concentration $(0.10 \mathrm{M})$ was suitable for accurate and concordant results.

Effect of variation in reaction temperature was studied. The reactivity of the sample is very slow at ice cold temperature but increases with the rise in temperature up to room temperature $\left(25-30{ }^{\circ} \mathrm{C}\right)$. Beyond this temperature, no improvement over the results has been noticed.

\section{Conclusion}

On the basis of available literature and stoichiometry established between antituberculous drugs and ammonium hexanitratocerate(IV) i.e., cycloserine (1:6), ethambutol (1:6), isoniazid (1:6), pyrazinamide (1:4) and rifampicin (1:4). This ratio remains constant even under varying reaction conditions i.e. change in reaction time, concentration of the reagent; reaction medium, etc.

\section{Interferences}

Excipients like starch, calcium carbonate, sodium carbonate, cellulose, magnesium trisilicate, tricalcium phosphate and gum acacia if present in the pharmaceutical preparations do of tablets and other excipients in injection not interfere in the estimation.

\section{ACKNOWLEDGEMENTS}

The authors are greatly thankful to Zydus Cadila, Cadila Healthcare Ltd. Ahmedabad and Lupin Ltd. Aurangabad, India for providing reference substance of antituberculous drugs as a gift. The authors Vinod Kumar and Brijesh K. Singh are also thankful to University Grant Commission, New Delhi, India for providing financial assistance in the form of research fellowship.

\section{REFERENCES}

1. J.R. Hwu and K.Y. King, Curr. Sci., 81, 1043 (2001).

2. M.-I. Lannou, F. Hélion and J.-L. Namy, Tetrahedron, 59, 10551 (2003).

3. K. Basavaiah, V. Ramakrishna and U.R.A. Kumar, Acta Pharm., 57, 211 (2007).

4. I.B. Sharma, V. Singh and M. Lakhanpal, J. Thermal Anal., 38, 1345 (1992).

5. L. Haiping, L. Ning and B. Sifu, J. Rare Earths, 24, 180 (2006).

6. L.G. Chatten, R.A. Locock and R.D. Krause, J. Pharm. Sci., 60, 588 (1971).

7. I. Miguel-Garcia, S. Parres-Esclapez, D. Lozano-Castello and A. BuenoLopez, Catal. Commun., 11, 848 (2010).

8. A.K. Dorai, S. Selvasekarapandian, N. Hellar, S. Ayyasamy and H. Muthusamy, Ionics, 16, 481 (2010).

9. F.J. Wegehaupt, B. Sener, T. Attin and P.R. Schmidlin, Arch. Oral Biol., 55, 441 (2010).

10. D.A. Conlon, D. Kumke, C. Moeder, M. Hardiman, G. Hutson and L. Sailer, Adv. Synth. Catal., 346, 1307 (2004).

11. C.J. Shih, Y.J. Chen and M.H. Hon, Mater. Chem. Phys., 121, 99 (2010).

12. E.G. Heckert, A.S. Karakoti, S. Seal and W.T. Self, Biomaterials, 29, 2705 (2008).

13. M.A. Arenas, A. Conde and J.J.D. Damborenea, Corr. Sci., 44, 511 (2002).

14. M. Wu, Q. Zhang, Y. Liu, Q. Fang and X. Liu, Mater. Res. Bull., 44, 1437 (2009).

15. T. Arii, T. Taguchi, A. Kishi, M. Ogawa and Y. Sawada, J. Eur. Ceram. Soc., 22, 2283 (2002).

16. Indian Pharmacopoeia, Controller of Publication, Govt. of India, Ministry of Health and Family Walfare, New Delhi (2008). 